\title{
Identification of HLA-A24-Restricted Novel T Cell Epitope Peptides Derived from P-Cadherin and Kinesin Family Member 20A
}

\author{
Ryuji Osawa, ${ }^{1,2}$ Takuya Tsunoda, ${ }^{1,2,3}$ Sachiko Yoshimura, ${ }^{1,2}$ \\ Tomohisa Watanabe, ${ }^{2}$ Motoki Miyazawa, ${ }^{1}$ Masaji Tani, ${ }^{1}$ Kazuyoshi Takeda, ${ }^{4}$ \\ Hidewaki Nakagawa, ${ }^{3}$ Yusuke Nakamura, ${ }^{3}$ and Hiroki Yamaue ${ }^{1}$ \\ ${ }^{1}$ Second Department of Surgery, Wakayama Medical University, 811-1 Kimiidera, Wakayama 641-8510, Japan \\ ${ }^{2}$ OncoTherapy Science Inc. Research Department, Kanagawa 213-0012, Japan \\ ${ }^{3}$ Laboratory of Molecular Medicine, Human Genome Center, Institute of Medical Science, The University of Tokyo, \\ Tokyo 108-8639, Japan \\ ${ }^{4}$ Department of Immunology, Juntendo University School of Medicine, Tokyo 113-8421, Japan
}

Correspondence should be addressed to Hiroki Yamaue, yamaue-h@wakayama-med.ac.jp

Received 6 February 2012; Revised 9 April 2012; Accepted 9 April 2012

Academic Editor: Soldano Ferrone

Copyright (C) 2012 Ryuji Osawa et al. This is an open access article distributed under the Creative Commons Attribution License, which permits unrestricted use, distribution, and reproduction in any medium, provided the original work is properly cited.

\begin{abstract}
We here identified human leukocyte antigen-(HLA-)A*2402-restricted epitope peptides from Cadherin 3, type 1, P-cadherin (CDH3) and kinesin family member 20A (KIF20A) that were found to be specifically expressed in cancer cells through genomewide expression profile analysis. CDH3-10-807 peptide and KIF20A-10-66 peptide successfully induced specific CTL clones, and these selectively responded to COS7 cells expressing both HLA-A*2402 and respective protein while did not respond to parental cells or COS7 cells expressing either HLA-A* 2402 or respective protein. Furthermore, CTL clones responded to cancer cells that endogenously express HLA-A*2402 and respective protein, suggesting that CDH3-10-807 peptide and KIF20A-10-66 peptide are naturally presented on HLA-A*2402 molecule of human cancer cells. Our results demonstrated that CDH3-10-807 peptide and KIF20A-10-66 peptide are novel HLA-A24-restricted tumor-associated antigens and would be applicable for CTL-inducing cancer therapies.
\end{abstract}

\section{Introduction}

After identification of the melanoma antigen gene (MAGE) family as a tumor-associated antigen (TAA), a number of TAAs have been revealed by means of various approaches including SEREX and cDNA library screening [1-6]. Some TAAs, such as MAGE, gp100, and MUC1, have been applied to treat various cancers in clinical trials [7-9], and vaccinebased therapy is now considered as a promising approach to fight against various cancers [10-13].

We have identified dozens of genes specifically expressed in cancer cells by genome-wide expression profile analysis for cDNA microarray consisting of more than 30,000 cDNAs and expressed sequence tags (ESTs) [14]. Among them, two genes, Cadherin 3, type 1, P-cadherin (CDH3) and kinesin family member 20A (KIF20A), were found to be upregulated in pancreatic cancers $[15,16]$. $\mathrm{CDH} 3$ is one of the classic cadherin family that plays a critical role in cell-cell adhesion and epithelial morphogenesis [17]. We reported that overexpression of $\mathrm{CDH} 3$ promoted the motility of cancer cells and blocking of $\mathrm{CDH} 3$ by anti-CDH3 antibody inhibited the migration of CDH3-expressing cells [15]. KIF20A is a member of the kinesin family, which is characterized to be a motor protein in cancer cells [18], and northern analysis indicated no expression of KIF20A among examined 23 normal tissues except testis. Furthermore, knock down of KIF20A expression with small interfering RNA suppressed the proliferation of pancreatic ductal adenocarcinoma cells [16]. 
Thus, both $\mathrm{CDH} 3$ and KIF20A would play oncogenic functions in pancreatic cancer cells and are attractive target molecules for cancer therapies including immunotherapy.

We here identified CDH3- and KIF20A-derived novel HLA-A*2402-restricted epitope peptides that can induce peptide-specific cytotoxic T lymphocyte (CTL), suggesting that these epitope peptide would be applicable to peptidebased cancer vaccine therapies for HLA-A*2402 positive pancreatic cancer patients.

\section{Materials and Methods}

2.1. Peptides. $\mathrm{CDH} 3$ and KIF20A-derived 9-mer and 10-mer peptides that have high binding affinity (binding score $>10$ ) to HLA-A* 2402 were predicted by the binding prediction software "BIMAS" (http://www-bimas.cit.nih.gov/molbio/ hla_bind/) and were synthesized by Sigma-Aldrich Japan KK (Ishikari, Japan) according to a standard solid-phase synthesis method and purified by reversed-phase high-performance liquid chromatography (HPLC). HIV-A24 epitope peptide (RYLRDQQLL) [19] was also synthesized as a negative control. The purity $(>90 \%)$ and the identity of the peptides were confirmed by analytical HPLC and mass spectrometry analysis, respectively. Peptides were dissolved in dimethylsulfoxide at $20 \mathrm{mg} / \mathrm{mL}$ and stored at $-80^{\circ} \mathrm{C}$.

2.2. Cell Lines. CDH3- and KIF20A- negative Human Blymphoblastoid cell line TISI (HLA-A*2402) was purchased from the IHWG Cell and Gene Bank (Seattle, WA). Monkey kidney cell line COS7, human B-lymphoblastoid cell line Jiyoye (HLA-A32), human B-lymphoblastoid cell line EB-3 (HLA-A3/Aw32), and CDH3-expressing human lung cancer cell line H358 (HLA-A3) were purchased from American Type Culture Collection (Manassas, VA). CDH3-expressing human pancreatic cancer cell line PK-45P (HLA-A24/A33) and KIF20A-expressing human pancreatic cancer cell line PK-59 (HLA-A31/A33) were provided by Cell Resource Center for Biomedical Research, Tohoku University (Sendai, Japan). KIF20A-expressing human stomach cancer cell line MKN-45 (HLA-A24) and MiaPaCa-2 cells (HLA-A24) were purchased from Health Science Research Resources Bank (Osaka, Japan). TISI, Jiyoye, EB-3, H358, PK-45P, PK59, and MKN-45 were maintained in RPMI1640 media (Invitrogen, Carlsbad, CA), COS7 were maintained in DMEM media (Invitrogen), and MiaPaCa-2 cells were maintained in EMEM media (Invitrogen). Each medium was supplemented with $10 \%$ fetal bovine serum (GEMINI BioProducts, West Sacramento, CA) and 1\% antibiotic solution (Sigma-Aldrich, ST. Louis, MO). The expression of CDH3 and KIF20A protein was confirmed by Western blotting using anti-CDH3 antibody (BD Transduction Labs., BD Biosciences, San Jose, CA) or anti-KIF20A antibody (Bethyl Laboratories, Montgomery, TX).

2.3. In Vitro Induction of Peptide-Specific CTL. To examine the ability to induce peptide-specific CTL, purified $\mathrm{CD} 8^{+}$ $\mathrm{T}$ cells were cocultured with autologous monocyte-derived mature dendritic cells (DCs) pulsed with peptide. Both
$\mathrm{CD}^{+} \mathrm{T}$ cells and DCs were prepared from peripheral blood mononuclear cells (PBMCs) of same HLA-A*2402-positive healthy volunteers. Briefly, PBMCs were isolated by FicollPaque solution (GE Healthcare, Uppsala, Sweden), then cells were cultured in AIM-V medium (Invitrogen) containing $2 \%$ heat-inactivated autologous serum (AS). After the over night incubation, nonadherent cells were washed out, then $1000 \mathrm{U} / \mathrm{mL}$ of granulocyte-macrophage colony-stimulating factor (GM-CSF; R\&D Systems, Minneapolis, MN) and $1000 \mathrm{U} / \mathrm{mL}$ of interleukin (IL)-4 (R\&D Systems) were added in the culture to induce monocyte-derived DCs. To mature DCs, $0.1 \mathrm{KE} / \mathrm{mL}$ of OK-432 (Chugai Pharmaceutical Co., Tokyo, Japan) was added in the culture on day 5 . Seven days later, DCs were pulsed with $20 \mu \mathrm{g} / \mathrm{mL}$ of synthesized peptide in AIM-V medium containing $3 \mu \mathrm{g} / \mathrm{mL}$ of $\beta 2$-microglobulin (Sigma-Aldrich) at $37^{\circ} \mathrm{C}$ for $3 \mathrm{~h}[20]$ and incubated in the media containing $30 \mu \mathrm{g} / \mathrm{mL}$ of Mitomycin C (MMC) (Kyowa Hakko Kirin Co. Ltd., Tokyo, Japan) for $30 \mathrm{~min}$. Following washing out residual peptide and MMC, cells were used as antigen-presenting cells to induce peptide-specific CTL. Generated monocyte-derived mature DCs expressed CD80, CD83, CD86, and HLA class II on their cell surface (data not shown). Autologous $\mathrm{CD}^{+} \mathrm{T}$ cells were prepared from PBMCs derived from the same HLA-A*2402-positive donor by positive selection with Dynal CD8 positive isolation kit (Invitrogen) according to the manufacturer's instructions. $1.5 \times 10^{4}$ of peptide-pulsed DCs and $3 \times 10^{5}$ of $\mathrm{CD}^{+} \mathrm{T}$ cells were cocultured in $0.5 \mathrm{~mL}$ of AIM-V medium supplemented with $10 \mathrm{ng} / \mathrm{mL}$ of IL-7 (R\&D Systems) and 2\% AS on 48well plates (Corning Inc., Corning, CA). IL-2 (CHIRON, Emeryville, CA) was added to the culture at $20 \mathrm{IU} / \mathrm{mL} 3$ days after coculture, and peptide-pulsed DCs were additionally supplied into the culture on days 7 and 14. Eight wells were prepared for CTL induction by every peptide in a single experiment. On day 21 , interferon- (IFN-) $\gamma$ production was examined by IFN- $\gamma$ enzyme-linked immunospot (ELISPOT) assay under the stimulation with peptide-pulsed TISI cells.

2.4. IFN- $\gamma$ Enzyme-Linked Immunospot (ELISPOT) Assay. $\mathrm{T}$ cell response to epitope peptide was measured by ELISPOT assay using IFN- $\gamma$ ELISPOT kit and AEC substrate set (BD Pharmingen, San Diego, CA) according to the manufacturer's instruction. Briefly, TISI cells were pulsed with $20 \mu \mathrm{g} / \mathrm{mL}$ of respective peptide at $37^{\circ} \mathrm{C}$ for $20 \mathrm{~h}$, and the residual peptide that did not bind to TISI cells was washed out to prepare peptide-pulsed TISI cells as the stimulator cells. $200 \mu \mathrm{L}$ of cell culture suspension were distributed to two wells (100 $\mu \mathrm{L}$ each) on Multiscreen-IP 96-well plate (Millipore, Bedford, MA) following removing $500 \mu \mathrm{L}$ of supernatant from each well from culture of "in vitro induction of peptide-specific CTL." Cells were coincubated with peptide-pulsed TISI cells $\left(1 \times 10^{4}\right.$ cells/well $)$ at $37^{\circ} \mathrm{C}$ for $20 \mathrm{~h}$. The plates were analyzed by the automated ELISPOT reader, ImmunoSPOT S4 (Cellular Technology Ltd, Cleveland, $\mathrm{OH}$ ) and ImmunoSpot Professional Software Version 5.0 (Cellular Technology Ltd). TISI cells pulsed with HIV-A24 epitope peptide (RYLRQQLLGI) were used as control. When the spot number in the peptide-stimulating 


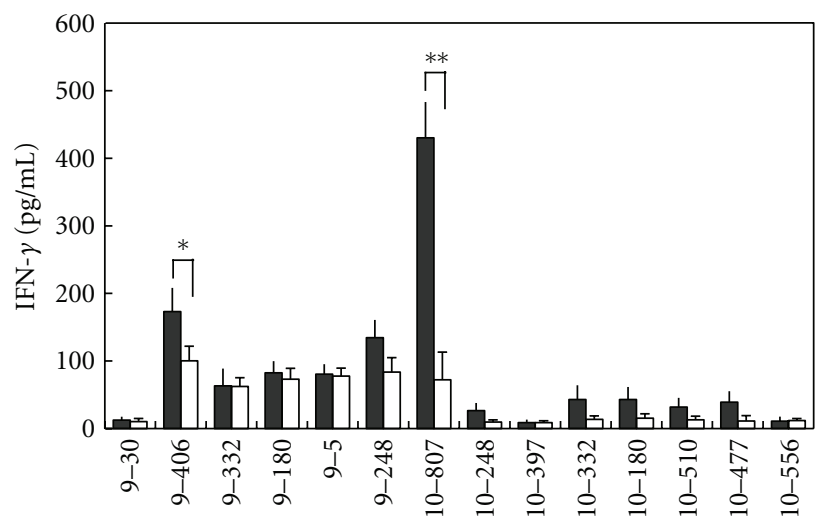

(a)

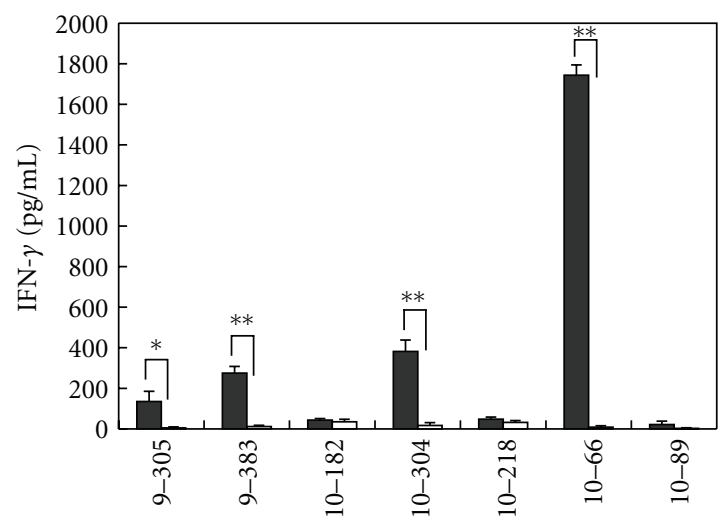

(b)

FIGURE 1: IFN- $\gamma$ production from CTLs responding to CDH3- or KIF20A-derived peptides. IFN- $\gamma$ production by CTLs induced with CDH3derived peptides (a) or KIF20A-derived peptides (b) responding to respective peptide-pulsed HLA-A*2402 positive TISI cells. CTLs were expanded and harvested following "in vitro induction of peptide-specific CTL," and IFN- $\gamma$ production was examined by IFN- $\gamma$ ELISA. "Closed bar" indicates the mean IFN- $\gamma$ production responding to TISI cells pulsed with indicated peptide, and "open bar" indicates the mean IFN- $\gamma$ production responding to TISI cells pulsed with HIV-A24 peptide (negative control). All experiments were performed triplicate. Similar results were obtained in three to five independent experiments. ${ }^{*} P<0.05,{ }^{* *} P<0.01$.

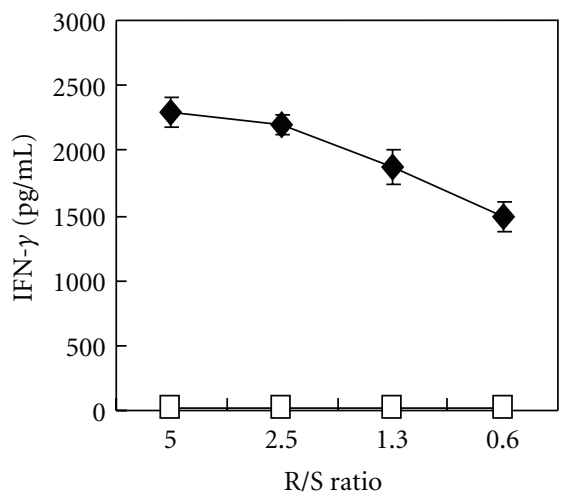

(a)

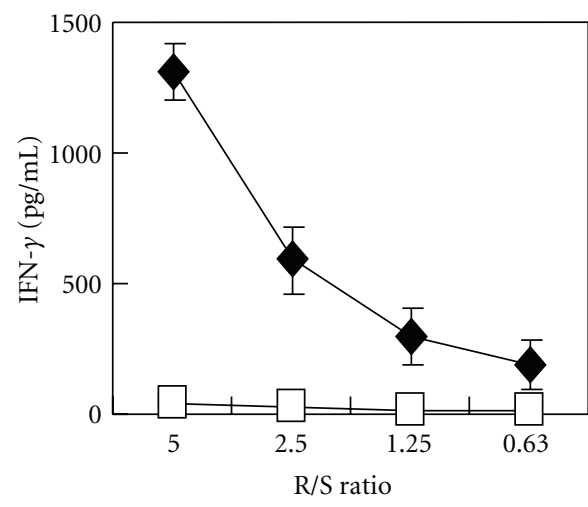

(c)

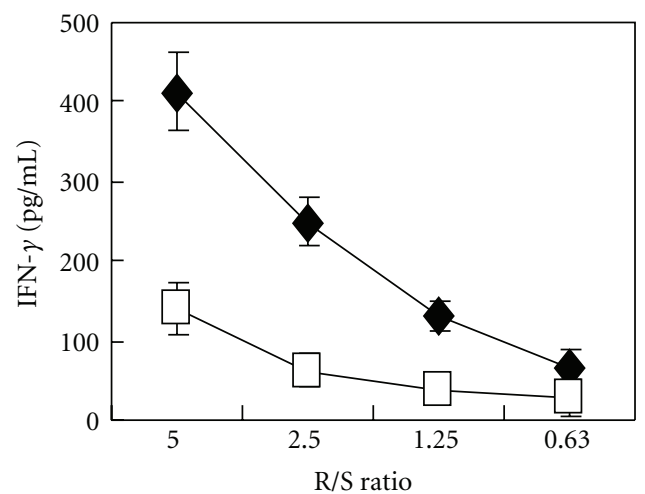

(b)

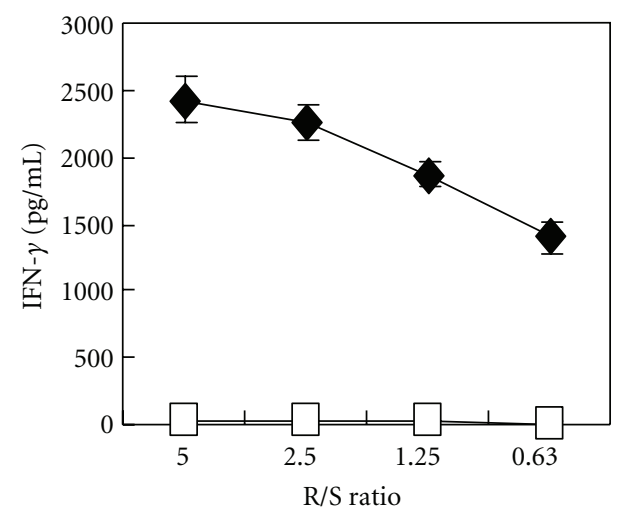

(d)

Figure 2: Peptide-specific IFN- $\gamma$ production by CTL clones. IFN- $\gamma$ production by CDH3-10-807 peptide-specific CTL clone (a), KIF20A-9305 peptide-specific CTL clone (b), KIF20A-10-304 peptide-specific CTL clone (c), and KIF20A-10-66 peptide-specific CTL clone (d), when stimulated with TISI cell pulsed with corresponding peptide (closed diamond) or HIV-A24 peptide (open square). CTL clones produced significant amount of IFN- $\gamma$ responding to corresponding peptide. IFN- $\gamma$ ELISA was performed triplicate. R/S ratio, responder cell (CTL clone)/stimulator cell (TISI cell) ratio. 
TABLE 1: Candidates of epitope peptide derived from CDH3 and KIF20A.

\begin{tabular}{|c|c|c|c|c|c|c|c|}
\hline \multicolumn{3}{|c|}{$\mathrm{CDH} 3$} & \multicolumn{5}{|c|}{ KIF20A } \\
\hline $\begin{array}{l}\text { Start } \\
\text { position }\end{array}$ & Amino acid sequence (mer) & $\begin{array}{l}\text { Binding } \\
\text { score }\end{array}$ & $\begin{array}{c}\text { CTL } \\
\text { induction }\end{array}$ & $\begin{array}{c}\text { Start } \\
\text { position }\end{array}$ & Amino acid sequence (mer) & $\begin{array}{l}\text { Binding } \\
\text { score }\end{array}$ & $\begin{array}{c}\text { CTL } \\
\text { induction }\end{array}$ \\
\hline 513 & IYEVMVLAM (9) & 37.5 & - & 308 & IYNELLYDL (9) & 432 & - \\
\hline 667 & LFLLLVLLL (9) & 36 & - & 621 & MYEEKLNIL (9) & 432 & - \\
\hline 30 & VFREAEVTL (9) & 24 & + & 67 & VYLRVRPLL (9) & 420 & - \\
\hline 406 & LYVEVTNEA (9) & 16.6 & + & 499 & KFSAIASQL (9) & 56 & - \\
\hline 332 & KYEAHVPEN (9) & 16.5 & + & 304 & SFFEIYNEL (9) & 44.352 & - \\
\hline 180 & KYELFGHAV (9) & 15 & + & 187 & IFNSLQGQL (9) & 36 & - \\
\hline 85 & RSLKERNPL (9) & 14.4 & - & 305 & FFEIYNELL (9) & 30 & + \\
\hline 5 & RGPLASLLL (9) & 12 & + & 23 & MFESTAADL (9) & 30 & - \\
\hline 652 & KGGFILPVL (9) & 11.2 & - & 256 & SFDSGIAGL (9) & 20 & - \\
\hline 248 & TYNGVVAYS (9) & 10.5 & + & 298 & RFSIWISFF (9) & 20 & - \\
\hline 65 & LFSTDNDDF (9) & 10 & - & 383 & IFSIRILHL (9) & 20 & + \\
\hline 807 & DYLNEWGSRF (10) & 150 & + & 647 & KIEELEALL (9) & 17.28 & - \\
\hline 248 & TYNGVVAYSI (10) & 105 & + & 625 & KLNILKESL (9) & 14.4 & - \\
\hline 667 & LFLLLVLLLL (10) & 42 & - & 695 & KLQQCKAEL (9) & 13.2 & - \\
\hline 397 & DFEAKNQHTL (10) & 30 & + & 726 & FTIDVDKKL (9) & 11.088 & - \\
\hline 332 & KYEAHVPENA (10) & 21 & + & 688 & QLQEVKAKL (9) & 11.088 & - \\
\hline 180 & KYELFGHAVS (10) & 15 & + & 308 & IYNELLYDLL (10) & 432 & - \\
\hline 510 & RNNIYEVMVL (10) & 12 & + & 182 & RSLALIFNSL (10) & 24.192 & + \\
\hline 5 & RGPLASLLLL (10) & 12 & - & 304 & SFFEIYNELL (10) & 24 & + \\
\hline 477 & RILRDPAGWL (10) & 12 & + & 742 & RLLRTELQKL (10) & 15.84 & - \\
\hline \multirow[t]{8}{*}{556} & CNQSPVRQVL (10) & 10.1 & + & 739 & KNIRLLRTEL (10) & 15.84 & - \\
\hline & & & & 218 & RQEEMKKLSL (10) & 14.4 & + \\
\hline & & & & 70 & RVRPLLPSEL (10) & 12.672 & - \\
\hline & & & & 871 & RILRSRRSPL (10) & 12 & - \\
\hline & & & & 89 & RIENVETLVL (10) & 12 & + \\
\hline & & & & 364 & KNQSFASTHL (10) & 12 & - \\
\hline & & & & 66 & KVYLRVRPLL (10) & 11.2 & + \\
\hline & & & & 60 & DSMEKVKVYL (10) & 10.08 & - \\
\hline
\end{tabular}

Start position indicated the number of amino acids from the $\mathrm{N}$ terminal of CDH3 and KIF20A.

Binding score was obtained using BIMAS program.

CTL induction was indicated as positive $(+)$ or negative $(-)$. Similar results were obtained 3-7 independent experiments using PBMC of 3-7 healthy volunteers.

well was more than 50 spots/well compared with that in the control well, we estimated that peptide-specific CTL were induced (positive) and subsequently expanded CTL from the positive well. Sensitivity of our ELISPOT assay was estimated as approximately average level by ELISPOT panel of Cancer Immunotherapy Consortium [CIC (http://www.cancerresearch.org/consortium/assay-panels/)].

2.5. CTL Expansion. Peptide-specific CTL obtained from CTL positive well of "in vitro induction of peptide-specific CTL" were expanded by the modified protocol based on the previously described methods [21-24]. Briefly, $5 \times 10^{5}$ of CTLs were cocultured with $5 \times 10^{6}$ of MMC-treated ( $30 \mu \mathrm{g} / \mathrm{mL}$ at $37^{\circ} \mathrm{C}$ for $30 \mathrm{~min}$ ) EB-3 and Jiyoye cells in $25 \mathrm{~mL}$ of AIM-V containing $5 \%$ AS and $40 \mathrm{ng} / \mathrm{mL}$ of anti-CD3 $\mathrm{mAb}$. The cultures were supplemented with IL-2 (final concentration: $120 \mathrm{IU} / \mathrm{mL}$ ) $24 \mathrm{~h}$ later and fed with AIM-V medium containing 5\% AS and IL-2 $(30 \mathrm{IU} / \mathrm{mL})$ on day 5,8 , and 11 . On day 14, expanded $\mathrm{T}$ cells were harvested to examine specific response to epitope peptide by IFN- $\gamma$ enzyme-linked immunosorbent assay (ELISA).
2.6. Establishment of Peptide-Specific CTL Clone. Peptidespecific CTL clones were established by limiting dilution method from the expanded CTLs specifically responding to epitope peptide. Briefly, T cells were diluted to $0.3,1$, and 3 cells/well in 96-well round-bottomed plates and cultured with $1 \times 10^{4}$ cells/well of MMC-treated EB-3 and Jiyoye cells in $150 \mu \mathrm{L}$ of AIM-V containing $5 \%$ AS, $125 \mathrm{IU} / \mathrm{mL}$ of IL-2, and $30 \mathrm{ng} / \mathrm{mL}$ of anti-CD3 mAb. The culture was supplemented with IL-2 to the final concentration of $125 \mathrm{IU} / \mathrm{mL}$ on day 10 . On day 14 , IFN- $\gamma$ production from peptidespecific CTL clones was examined by IFN- $\gamma$ ELISA. Some peptide-specific CTL clones were expanded as described above.

2.7. IFN- $\gamma$ ELISA. In some experiments, established CTLs were co-incubated with $1 \times 10^{4}$ cells of respective peptidepulsed TISI cells or $5 \times 10^{4}$ cells of COS7 cells in $200 \mu \mathrm{L}$ of AIM-V/5\% AS media on 96-well round bottom plate (Corning Inc.). After $24 \mathrm{~h}$ incubation, cell free supernatants were harvested and IFN- $\gamma$ production was examined by human 


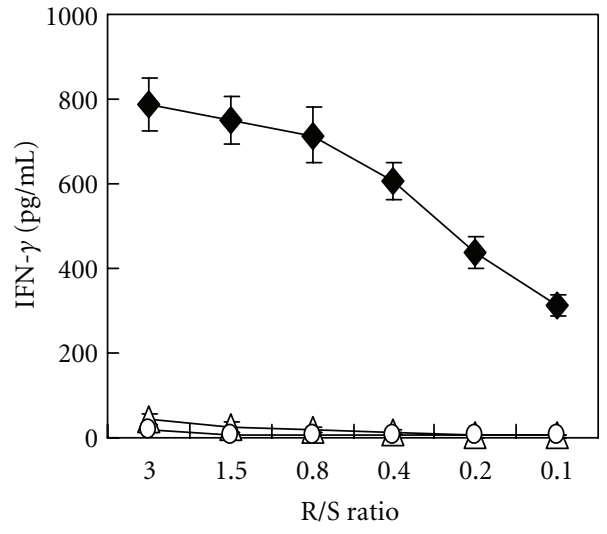

(a)

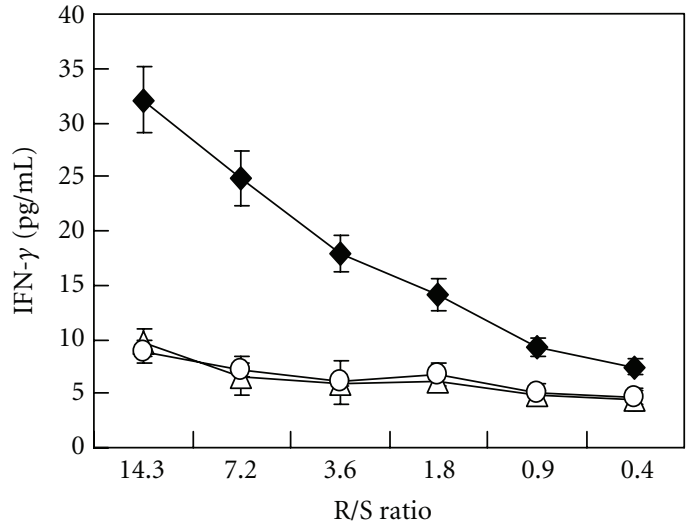

(b)

FIGURE 3: IFN- $\gamma$ production by CTL clones responding to COS7 cells that expressing HLA-A*2402 and respective oncogene. (a) IFN- $\gamma$ production by CDH3-10-807 peptide-specific CTL clone when exposed with COS7 cells expressing both HLA-A*2402 and CDH3 (closed diamond), HLA-A*2402 (open triangle), or CDH3 (open circle). (b) IFN- $\gamma$ production by KIF20A-10-66 peptide-specific CTL clone when exposed with COS7 cells expressing HLA-A*2402 and KIF20A (closed diamond), HLA-A*2402 (open triangle), or KIF20A (open circle). Both CTL clones significantly produced IFN $-\gamma$ responding to COS7 cells expressing HLA-A*2402 and corresponding gene. Similar results were obtained in three independent experiments. Independently induced other CTL clones also produced significant amount of IFN- $\gamma$ when exposed with COS7 cell expressing both HLA-A*2402 and respective gene (data not shown). R/S ratio, responder cell (CTL clone)/stimulator cell (COS7 cell) ratio.

IFN- $\gamma$-specific ELISA kit (BD Pharmingen) according to the manufacturer's instructions.

2.8. Cytotoxicity Assay. Specific cytotoxic activity of induced CTL clones was tested by a $4 \mathrm{~h}{ }^{51} \mathrm{Cr}$ release assay as previously described [25]. Data are represented as the mean \pm SD of triplicate samples.

2.9. Transfection of HLA-A24 and/or Oncogene (CDH3 or KIF20A). HLA-A*2402 coding region was obtained from TISI cells. The cDNA encoding an open reading frame of HLA-A*2402 gene with FLAG tag or oncogene (CDH3 or KIF20A) coding region with the Myc tag sequence was amplified with PCR and cloned into pcDNA3.1 vector (Invitrogen). COS7 cells transiently expressing HLA-A*2402 and/or oncogene were prepared by the transfection of the vectors encoding respective genes using lipofectamine 2000 (Invitrogen) according to the manufacturer's instruction. The expression of HLA-A*2402 and oncogene-derived protein was confirmed by Western blotting using anti-Myc (Upstate Biotechnology, Lake Placid, NY) or anti-FLAG antibody (Sigma-Aldrich). Two days after transfection, the transfected cells were harvested with versene (Invitrogen) and used to stimulate peptide-specific CTL clones. IFN- $\gamma$ production by CTLs was examined by IFN- $\gamma$-specific ELISA.

2.10. Flow Cytometry. Expression of peptide-specific T cell receptor (TCR) was examined on FACS-CantoII (Becton Dickinson, San Jose, CA) using peptide-HLA-A*2402 dextramer-PE (Immudex, Copenhagen, Denmark) (CDH310-807/MHC-dextramer-PE and KIF20A-10-66/MHCdextramer-PE) according to the manufacturer's instructions. HIV-A24 epitope peptide (RYLRDQQLL)/MHC-dextramer was used as negative control. Briefly, expanded CTL lines were incubated with peptide-HLA-A*2402 dextramer-PE for 10 minutes at room temperature, then treated with FITCconjugated anti-human CD8 mAb, APC-conjugated anti-human CD3 mAb, PE-Cy7-conjugated anti-human $\mathrm{CD} 4 \mathrm{mAb}$, and 7-AAD (BD Pharmingen) at $4^{\circ} \mathrm{C}$ for 20 minutes.

\section{Results}

3.1. Induction of CTL Responding to CDH3- or KIF20ADerived Peptide Restricted with HLA-A*2402. Based on the analysis with the binding prediction software "BIMAS," we synthesized $21 \mathrm{CDH} 3$-derived epitope-peptides and 28 KIF20A-derived epitope-peptides that were expected to have high affinity to HLA-A*2402 molecule and activate CTLs (Table 1).

HLA-A*2402-positive $\mathrm{CD}^{+} \mathrm{T}$ cells were cocultured with autologous DCs pulsed with respective peptide, and then peptide-specific IFN- $\gamma$ production was analyzed by ELISPOT. Fourteen peptides derived from $\mathrm{CDH} 3$ and 7 peptides derived from KIF20A were able to induce peptidespecific CTLs producing IFN- $\gamma$ (Table 1). Amongst these peptides, we successfully obtained CTLs that specifically produced significant amount of IFN- $\gamma$ after CTL expansion when CDH3-9-406, CDH3-10-807, KIF20A-9-305, KIF20A9-383, KIF20A-10-304, and KIF20A-10-66 peptide were pulsed (Figures 1(a) and 1(b)).

3.2. Establishment of CDH3- or KIF20A-Derived PeptideSpecific CTL Clones. Subsequently, we attempted to establish CTL clones by a limiting dilution. CDH3-10-807-, KIF20A9-305-, KIF20A-10-304-, or KIF20A-10-66-specific CTL clones were established and produced a potent amount of 

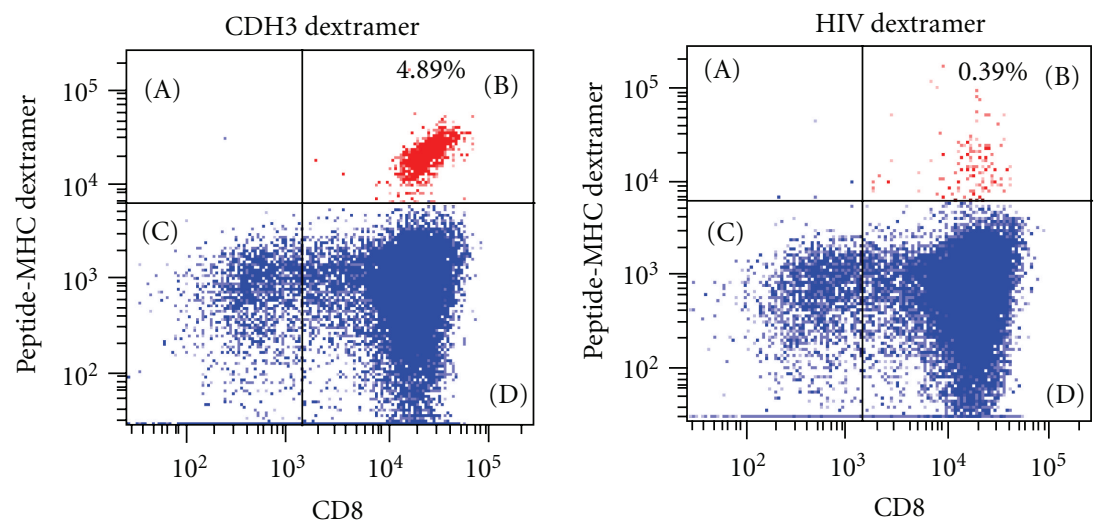

(a)
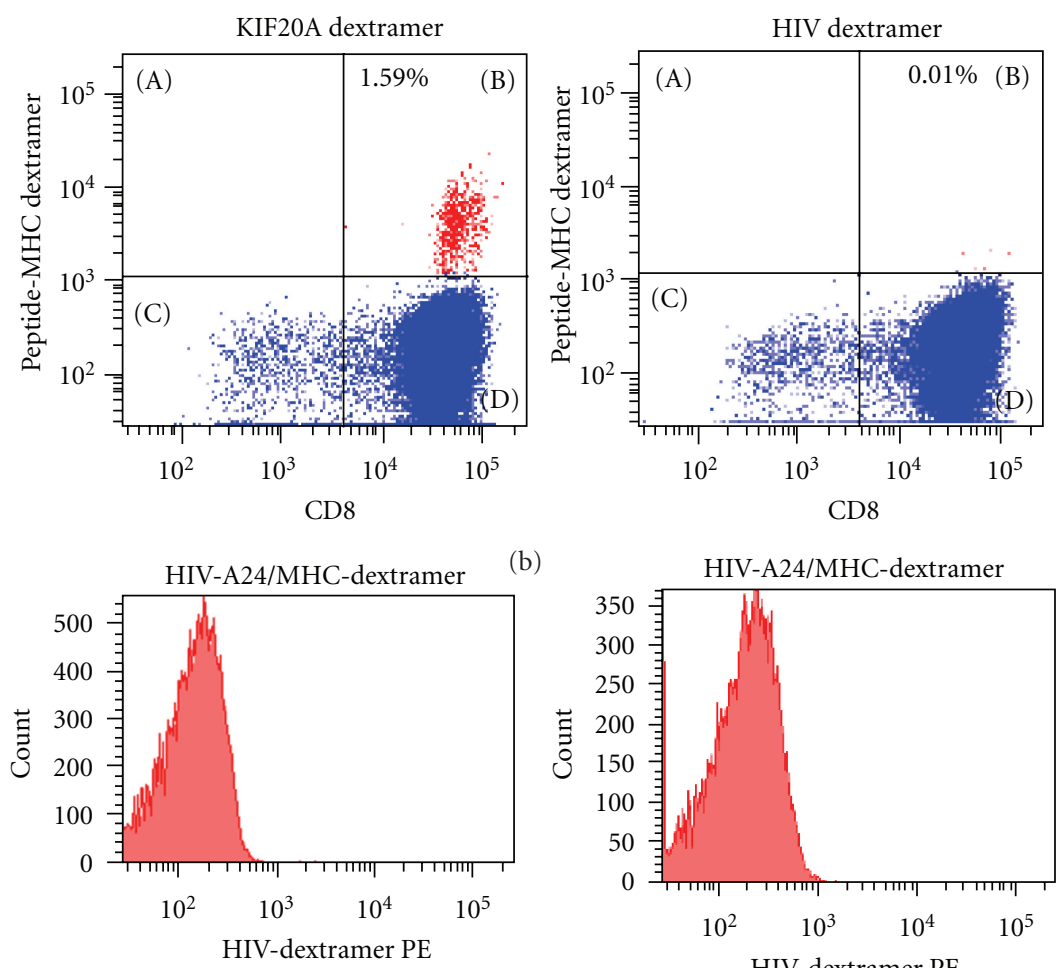

(b)

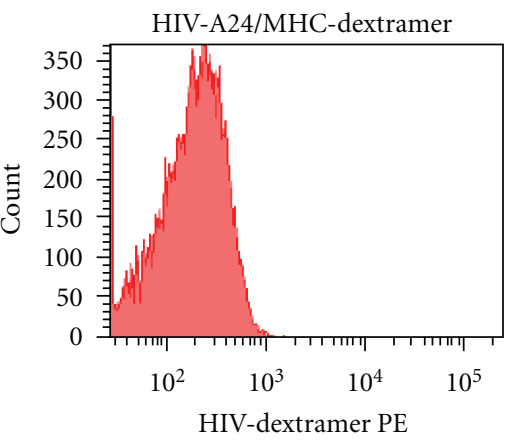

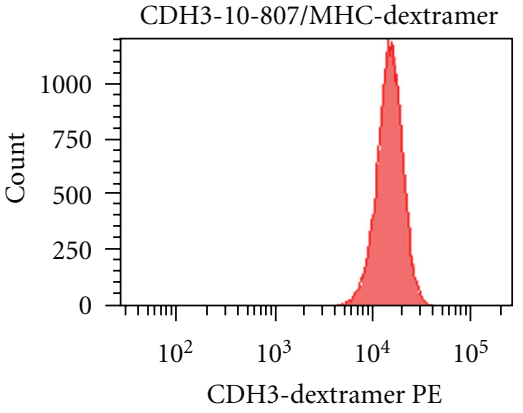

(c)

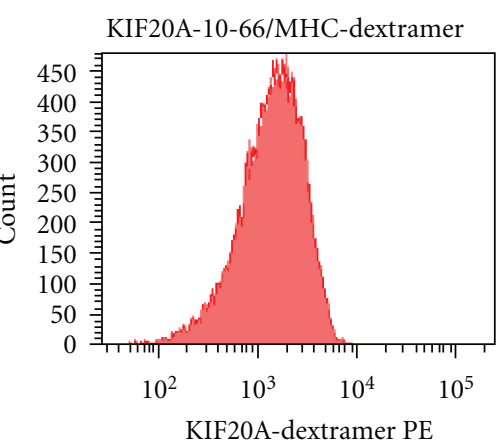

(d)

Figure 4: Peptide-specific TCR expression on CTL clones. (a) CDH3-10-807/HLA-A*2402-specific TCR expressing cells in expanded CTLs following "in vitro induction of peptide-specific CTL." (b) KIF20A-10-66/HLA-A*2402-specific TCR expressing cells in expanded CTLs following "in vitro induction of peptide-specific CTL." Results staining with anti-human CD8 mAb and CDH3-10-807/MHC-dextramer-PE or KIF20A-10-66/MHC-dextramer-PE are presented following gating on CD3-positive cells (left panels). Results staining with anti-human $\mathrm{CD} 8 \mathrm{mAb}$ and HIV-A24/MHC-dextramer-PE are presented as negative control following gating on CD3 positive cells (right panels). (c) CDH3-10-807/HLA-A*2402-specific TCR expression on CDH3-10-807-specific CTL clone. (d) KIF20A-10-66/HLA-A*2402-specific TCR expression on KIF20A-10-66-specific CTL clone. Staining with HIV-A24/MHC-dextramer-PE was used as negative control. CTL clones were $\mathrm{CD}^{+}$and $\mathrm{CD}^{+}$as expected (data not shown). Similar results were obtained in independent all experiments to examine CTL induction. 


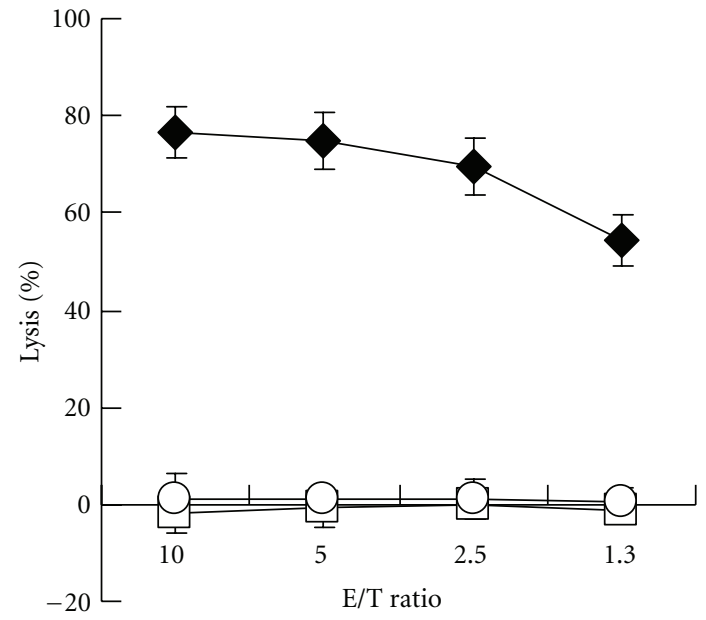

(a)

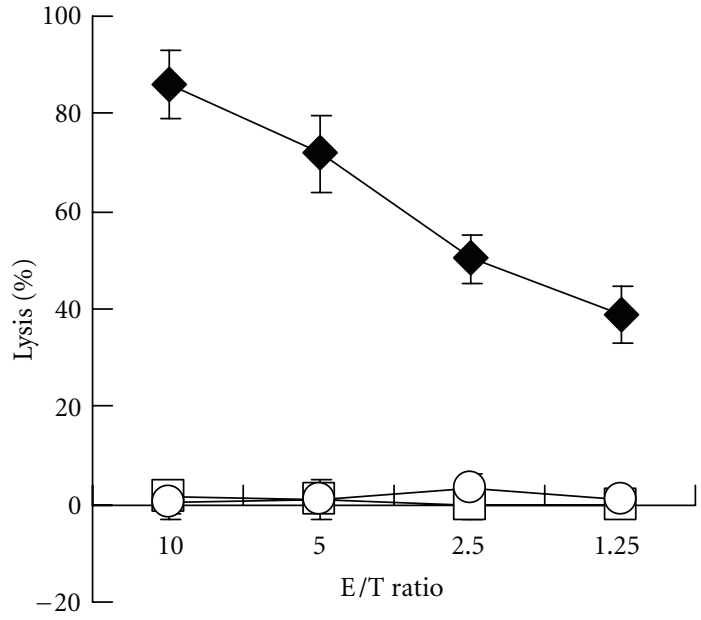

(b)

FIgUre 5: Cytotoxic activity of CTL clones against peptide-pulsed TISI cells. Cytotoxic activity of CDH3-10-807 peptide-specific CTL clone (a) and KIF20A-10-66 peptide-specific CTL clone (b) against HLA-A*2402-positive TISI cells pulsed with respective peptide (close diamond), HIV-A24 peptide (open square), or TISI cells without peptide pulse (open circle). E/T ratio, effector cell (CTL clone)/target cell (TISI cell) ratio. Similar results were obtained in three independent experiments using same CTL clone and in independent experiments using other CTL clones.

IFN- $\gamma$ specifically responding to the stimulator cells pulsed with the respective peptide, but not HIV-A24 peptide (Figures 2(a)-2(d)).

3.3. Recognition of Cells Endogenously Expressing Both HLA$A^{*} 2402$ and Respective Protein by Peptide-Specific CTL Clones. We then examined that the established peptide-specific CTL clones can recognize cells that express HLA-A*2402 and the target proteins. COS7 cells were transfected with plasmid designed to express HLA-A*2402 molecule and/or that to express the full length protein of $\mathrm{CDH} 3$ or KIF20A. We confirmed expression of these proteins by western blotting (data not shown). CDH3-10-807 peptide responding CTL clone substantially produced IFN- $\gamma$ when exposed to COS7 cells that expressing both HLA-A*2402 and CDH3, but not COS7 cells that expressing either HLA-A*2402 or CDH3 (Figure 3(a)). Similarly, KIF20A-10-66 peptide responding CTL clone produced significant amount of IFN- $\gamma$ when exposed to COS7 cells that expressing both HLA-A*2402 and KIF20A, but not COS7 cells that expressing either HLA-A*2402 or KIF20A (Figure 3(b)). Both CTL clones also produced IFN- $\gamma$ responding to COS7 cells, which transfected with pIRES-vector containing both HLA-A*2402 and respective oncogene (data not shown). On the other hand, CTL clones responding to KIF20A-9-305 peptide or KIF20A-10-304 peptide did not produce IFN- $\gamma$ when exposed to COS7 cells expressing both HLA-A*2402 and KIF20A (data not shown). Only CDH3-10-807 peptide and KIF20A-10-66 peptide, but not other candidate peptides, were able to induce CTL responding to COS7 cells expressing HLA-A*2402 and CDH3 or KIF20A, albeit we have tried several times using PBMC derived from different healthy donors (data not shown).
3.4. Peptide-Specific T Cell Receptor Expression. Expression of CDH3-10-807/HLA-A*2402- or KIF20A-10-66/HLA$\mathrm{A}^{*}$ 2402-specific $\mathrm{T}$ cell receptor (TCR) was examined using CDH3-10-807/MHC-dextramer-PE or KIF20A-10$66 / \mathrm{MHC}^{-d e x t r a m e r-P E}$. Significant population of $\mathrm{CD}^{+}$ $\mathrm{CD}^{+}$cells, but not $\mathrm{CD}^{+}{ }^{+} \mathrm{CD} 8^{-}$cells, expressed $\mathrm{CDH} 3-10-$ 807/HLA-A*2402- or KIF20A-10-66/HLA-A*2402- specific TCR after expansion of cells obtained by "in vitro induction of peptide-specific CTL" (Figures 4(a) and 4(b)). As expected, CTL clones established by CDH3-10-807 peptideor KIF20A-10-66 peptide-pulsed cells were CD8 positive and expressed respective peptide/HLA-A*2402-specific TCR (Figures 4(c) and 4(d)).

3.5. Cytotoxic Activity of CTLs. We also examined cytotoxic activity of CTL clones. CDH3-10-807 or KIF20A-10-66 peptide-specific CTL clone demonstrated cytotoxic activity against HLA-A*2402-positive TISI cells when respective peptide was pulsed, but not when HIV-A24 peptide was pulsed or peptide was not pulsed (Figures 5(a) and 5(b)). These results suggested that CDH3-10-807 or KIF20A-1066 peptide-specific CTL clone specifically exerted cytotoxic activity responding to respective epitope peptide binding to HLA-A*2402 on cells.

We, then, finally examined the cytotoxic activity against cancer cells, which endogenously expressed CDH3 or KIF20A gene. Expression of $\mathrm{CDH} 3$ protein was confirmed in HLA-A*2402-positive PK-45P cells and HLA-A*2402negative $\mathrm{H} 358$ cells, but HLA-A*2402-positive MiaPaca2 cells did not express CDH3 (Figure 6(a)). CDH3-10807 peptide-specific CTL clone exerted significant cytotoxic activity against $\mathrm{CDH} 3$-expressing HLA-A*2402-positive $\mathrm{PK}$ $45 \mathrm{P}$ cells, but not $\mathrm{H} 358$ or MiaPaca-2 cells (Figure 6(b)). 


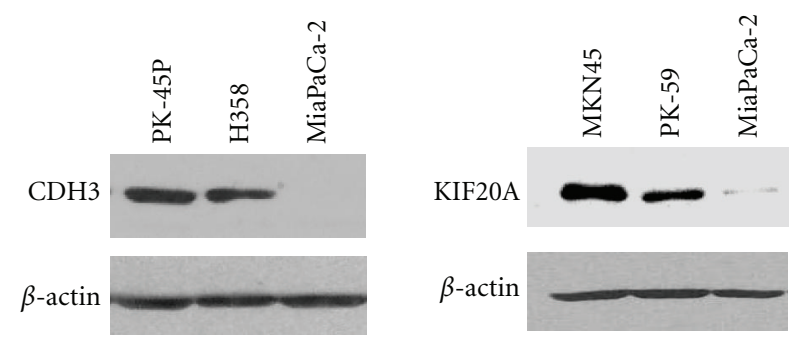

(a)

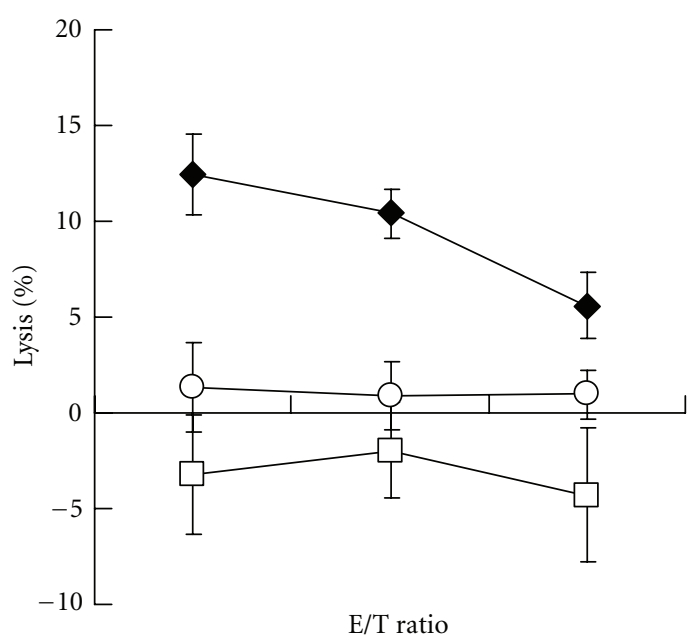

(b)

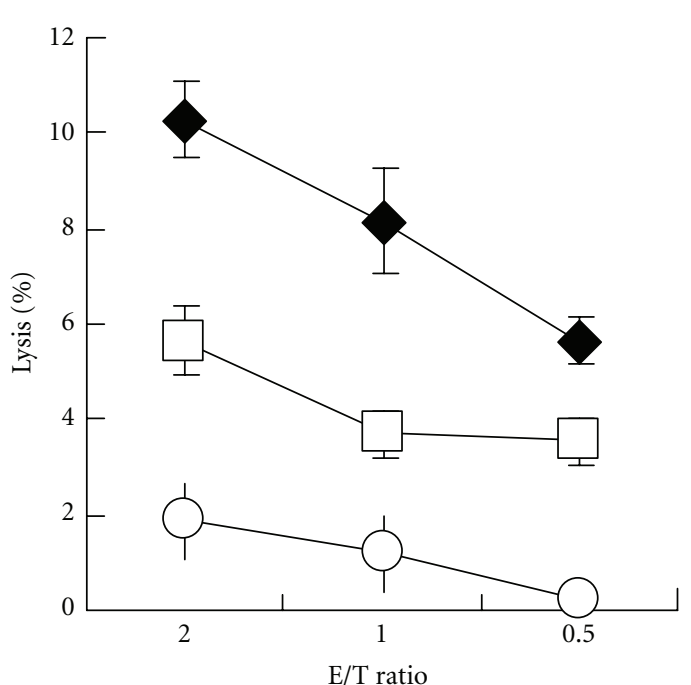

(c)

FIGURE 6: Cytotoxic activity against tumor cells expressing respective gene. (a) The expression of CDH3 and KIF20A protein in tumor cells used in cytotoxic assay. (b) Cytotoxic activity of CDH3-10-807 peptide-specific CTL clone against CDH3-expressing HLA-A*2402positive PK-45P cells (closed diamond), CDH3-expressing HLA-A*2402-negative H358 cells (open square), or MiaPaCa-2 cells (open circle). Cytotoxicity against PK-45P cells was significantly higher than those against other cells. (c) Cytotoxic activity of KIF20A-10-66 peptidespecific CTL clone against KIF20A-expressing HLA-A*2402-positive MKN-45 cells (closed diamond), KIF20A-expressing HLA-A*2402negative PK-59 cells (open square), or MiaPaCa-2 cells (open circle). Cytotoxicity against MKN-45 cells or PK-59 cells are significantly higher than that against MiaPaCa-2 cells, although cytotoxic activity against PK-59 cells was significantly lower compared with that against MKN45 cells. E/T ratio, effector cell (CTL clone)/target cell (tumor cell) ratio. Similar results were obtained in three independent experiments using same CTL clone and in independent experiments using other CTL clones.

Expression of KIF20A protein was confirmed in HLAA*2402-positive MKN-45 cells and HLA-A*2402-negative PK-59 cells, but HLA-A*2402-positive MiaPaca-2 cells did not express KIF20A (Figure 6(a)). KIF20A-10-66 peptidespecific CTL clone exerted significant cytotoxic activity against KIF20A-expressing HLA-A*2402-positive MKN-45 cells, but not MiaPaca-2 cells (Figure 6(c)). KIF20A-10-66 peptide-specific CTL clone demonstrated cytotoxic activity against KIF20A-expressing HLA-A*2402-negative PK-59 cells; however, this cytotoxicity was always less when compared with that against KIF20A-expressing HLA-A*2402positive MKN-45 cells (Figure 6(c) and data not shown).

No homologous sequence to $\mathrm{CDH} 3-10-807$ peptide or KIF20A-10-66 peptide was demonstrated by the homology research using the BLAST algorithm http://blast.ncbi.nlm .nih.gov/Blast.cgi (data not shown), suggesting that these peptide would be the unique epitope peptide presented on HLA-A*2402 of CDH3 or KIF20A-expressing cells.

Taken together, presented results suggested that $\mathrm{CDH} 3-$ 10-807 peptide-specific or KIF20A-10-66 peptide-specific
CTLs exert potent IFN- $\gamma$ production and cytotoxic activity specifically responding to HLA-A*2402-positive cancer cells expressing $\mathrm{CDH} 3$ or KIF20A, respectively.

\section{Discussion}

Pancreatic cancer is one of the most malignant cancers, since 5 -year survival rate is only $5 \%$ and the therapeutic modalities are very limited $[26,27]$. Both $\mathrm{CDH} 3$ and KIF20A were upregulated in the majority of pancreatic cancers and have oncogenic functions $[15,16]$. Thus, $\mathrm{CDH} 3$ and KIF20A would be promising target molecules to develop novel therapeutic strategies for pancreatic cancer. Hence, we identified HLA-A*0201-restricted peptides derived from $\mathrm{CDH} 3$ and KIF20A $[28,29]$.

In present study, we successfully identified HLA-A*2402restricted novel epitope peptides derived from both $\mathrm{CDH} 3$ and KIF20A and demonstrated that these peptides could induce specific CTLs producing potent amount of IFN- $\gamma$ and 
exert cytotoxic activity. Established CDH3-10-807-specific CTL clones or KIF20A-10-66-specific CTL clones responded to $\mathrm{CDH} 3$ - or KIF20A-introduced COS7 cells as well as CDH3 or KIF20A endogenously expressing cancer cells (PK-45P or MKN-45) in HLA-A24-restricted manner. These results indicated that induction of CDH3-10-807-specific CTLs or KIF20A-10-66-specific CTLs would exert antitumor effect against pancreatic cancers in HLA-A24-positive patients.

Predicted binding score of KIF20A-10-66 peptide to HLA-A*2402 was relatively low when compared with that of CDH3-10-807 peptide. We previously reported epitope peptides derived from RNF43 and IMP-3, and those peptides also have low affinity to HLA molecule [22, 24]. Interestingly, both peptides have been already applied for clinical trials as peptide-based immunotherapy and CTL were obtained in many cancer patients $[11,12]$. These results suggested that some peptides possibly induce CTLs albeit binding score was low by BIMAS prediction and KIF20A-10-66 peptide, as well as CDH3-10-807 peptide, possibly induces CTL in cancer patients.

Recent improvement and development of cancer therapies, including combined treatments of standard therapies (chemotherapy, radiotherapy, and surgical resection), substantially improved the survival of advanced cancer patients [27]. However, unfavorable adverse events are still often observed. On the other hand, immune therapies inducing cancer-cell-specific CTLs are now developed to improve the efficacy against cancers and the quality of life of patients. Ongoing several clinical trials using epitope peptides derived from TAA have been proving the evidence that CTL-inducing therapies are much less harmful to the patients [10-12]. However, efficacy of some vaccine therapy trials is still limited mainly due to the development of escaping variant cancer cells that lost targeted TAA expression during the treatment [30]. Therefore, it is generally thought that the therapeutic efficacy would be improved when the origin of vaccinated peptide is functionary essential molecule for cancer cell survival, proliferation, and/or motility. We have been screening epitope peptides derived from cancer-specific genes and reported several epitope peptides, which can elicit specific CTL responses [21-24]. Some of these peptides have been already applied for translational researches of multipeptide vaccine to treat esophageal cancer and colorectal cancer $[11,12]$. Moreover, multiple-antigen vaccine therapy was suggested to more effectively hinder escape mechanisms in the guidance from Food and Drug Administration (Guidance for Industry: Clinical Considerations for Therapeutic Cancer Vaccines). Thus, we believe that identification of CTL-inducible epitope peptides derived from several molecules that play critical roles in various types of cancer is important to develop multipeptide cocktail, and that resulted in the improvement of efficacy of CTL-inducing cancer therapies.

Presented results demonstrated that $\mathrm{CDH} 3-10-807$ peptide and KIF20A-10-66 peptide pulsed DCs induced specific CTL to possibly exert antitumor effect. The immunogenicity of CDH3-10-807 peptide and KIF20A-10-66 peptide should be examined in patients bearing these genes-expressing cancers, and we are now going to conduct clinical trials.

\section{References}

[1] T. Boon, "Tumor antigens recognized by cytolytic T lymphocytes: present perspectives for specific immunotherapy," International Journal of Cancer, vol. 54, no. 2, pp. 177-180, 1993.

[2] T. Boon and P. Van der Bruggen, "Human tumor antigens recognized by T lymphocytes," Journal of Experimental Medicine, vol. 183, no. 3, pp. 725-729, 1996.

[3] P. van der Bruggen, C. Traversari, P. Chomez et al., "A gene encoding an antigen recognized by cytolytic T lymphocytes on a human melanoma," Science, vol. 254, no. 5038, pp. 1643$1647,1991$.

[4] V. Brichard, A. Van Pel, T. Wolfel et al., "The tyrosinase gene codes for an antigen recognized by autologous cytolytic T lymphocytes on HLA-A2 melanomas," Journal of Experimental Medicine, vol. 178, no. 2, pp. 489-495, 1993.

[5] Y. Kawakami, S. Eliyahu, K. Sakaguchi et al., "Identification of the immunodominant peptides of the MART-1 human melanoma antigen recognized by the majority of HLA-A2restricted tumor infiltrating lymphocytes," Journal of Experimental Medicine, vol. 180, no. 1, pp. 347-352, 1994.

[6] Y. T. Chen, M. J. Scanlan, U. Sahin et al., "A testicular antigen aberrantly expressed in human cancers detected by autologous antibody screening," Proceedings of the National Academy of Sciences of the United States of America, vol. 94, no. 5, pp. 19141918, 1997.

[7] S. R. Reynolds, A. Zeleniuch-Jacquotte, R. L. Shapiro et al., "Vaccine-induced CD8 ${ }^{+}$T-cell responses to MAGE-3 correlate with clinical outcome in patients with melanoma," Clinical Cancer Research, vol. 9, no. 2, pp. 657-662, 2003.

[8] S. A. Rosenberg, J. C. Yang, D. J. Schwartzentruber et al., "Recombinant fowlpox viruses encoding the anchor-modified gp100 melanoma antigen can generate antitumor immune responses in patients with metastatic melanoma," Clinical Cancer Research, vol. 9, no. 8, pp. 2973-2980, 2003.

[9] G. Pecher, A. Häring, L. Kaiser, and E. Thiel, "Mucin gene (MUC1) transfected dendritic cells as vaccine: results of a phase I/II clinical trial," Cancer Immunology, Immunotherapy, vol. 51, no. 11-12, pp. 669-673, 2002.

[10] M. Miyazawa, R. Ohsawa, T. Tsunoda et al., "Phase I clinical trial using peptide vaccine for human vascular endothelial growth factor receptor 2 in combination with gemcitabine for patients with advanced pancreatic cancer," Cancer Science, vol. 101, no. 2, pp. 433-439, 2010.

[11] K. Kono, Y. Mizukami, Y. Daigo et al., "Vaccination with multiple peptides derived from novel cancer-testis antigens can induce specific T-cell responses and clinical responses in advanced esophageal cancer," Cancer Science, vol. 100, no. 8, pp. 1502-1509, 2009.

[12] K. Okuno, F. Sugiura, J. I. Hida et al., "Phase I clinical trial of a novel peptide vaccine in combination with UFT/LV for metastatic colorectal cancer," Experimental and Therapeutic Medicine, vol. 2, no. 1, pp. 73-79, 2011.

[13] M. Palmer, J. Parker, S. Modi et al., "Phase I study of the BLP25 (MUC1 peptide) liposomal vaccine for active specific immunotherapy in stage IIIB/IV non-small-cell lung cancer," Clinical Lung Cancer, vol. 3, no. 1, pp. 49-57, 2001.

[14] T. Nakamura, Y. Furukawa, H. Nakagawa et al., "Genomewide cDNA microarray analysis of gene expression profiles in pancreatic cancers using populations of tumor cells and normal ductal epithelial cells selected for purity by laser microdissection," Oncogene, vol. 23, no. 13, pp. 2385-2400, 2004. 
[15] K. Taniuchi, H. Nakagawa, M. Hosokawa et al., "Overexpressed $\mathrm{P}$-cadherin/CDH3 promotes motility of pancreatic cancer cells by interacting with p120ctn and activating Rhofamily GTPases," Cancer Research, vol. 65, no. 8, pp. 3092 3099, 2005.

[16] K. Taniuchi, H. Nakagawa, T. Nakamura et al., "Downregulation of RAB6KIFL/KIF20A, a kinesin involved with membrane trafficking of discs large homologue 5, can attenuate growth of pancreatic cancer cell," Cancer Research, vol. 65, no. 1, pp. 105-112, 2005.

[17] M. Takeichi, "The cadherins: cell-cell adhesion molecules controlling animal morphogenesis," Development, vol. 102, no. 4, pp. 639-655, 1988.

[18] F. Lai, A. A. Fernald, N. Zhao, and M. M. Le Beau, "cDNA cloning, expression pattern, genomic structure and chromosomal location of RAB6KIFL, a human kinesin-like gene," Gene, vol. 248, no. 1-2, pp. 117-125, 2000.

[19] Y. Ikeda-Moore, H. Tomiyama, K. Miwa et al., "Identification and characterization of multiple HLA-A24-restricted HIV-1 CTL epitopes: strong epitopes are derived from $\mathrm{V}$ regions of HIV-1," Journal of Immunology, vol. 159, no. 12, pp. 62426252, 1997.

[20] E. Celis, V. Tsai, C. Crimi et al., "Induction of anti-tumor cytotoxic T lymphocytes in normal humans using primary cultures and synthetic peptide epitopes," Proceedings of the National Academy of Sciences of the United States of America, vol. 91, no. 6, pp. 2105-2109, 1994.

[21] H. Ishizaki, T. Tsunoda, S. Wada, M. Yamauchi, M. Shibuya, and H. Tahara, "Inhibition of tumor growth with antiangiogenic cancer vaccine using epitope peptides derived from human vascular endothelial growth factor receptor 1," Clinical Cancer Research, vol. 12, no. 19, pp. 5841-5849, 2006.

[22] N. Uchida, T. Tsunoda, S. Wada, Y. Furukawa, Y. Nakamura, and H. Tahara, "Ring finger protein 43 as a new target for cancer immunotherapy," Clinical Cancer Research, vol. 10, no. 24, pp. 8577-8586, 2004.

[23] S. Wada, T. Tsunoda, T. Baba et al., "Rationale for antiangiogenic cancer therapy with vaccination using epitope peptides derived from human vascular endothelial growth factor receptor 2," Cancer Research, vol. 65, no. 11, pp. 4939-4946, 2005.

[24] T. Suda, T. Tsunoda, Y. Daigo, Y. Nakamura, and H. Tahara, "Identification of human leukocyte antigen-A24-restricted epitope peptides derived from gene products upregulated in lung and esophageal cancers as novel targets for immunotherapy," Cancer Science, vol. 98, no. 11, pp. 1803-1808, 2007.

[25] K. Takeda, N. Yamaguchi, H. Akiba et al., "Induction of tumor-specific T cell immunity by anti-DR5 antibody therapy," Journal of Experimental Medicine, vol. 199, no. 4, pp. 437-448, 2004.

[26] A. Sultana, C. Tudur Smith, D. Cunningham et al., "Systematic review, including meta-analyses, on the management of locally advanced pancreatic cancer using radiation/combined modality therapy," British Journal of Cancer, vol. 96, no. 8, pp. 1183-1190, 2007.

[27] A. Jemal, R. Siegel, E. Ward, Y. Hao, J. Xu, and M. J. Thun, "Cancer statistics, 2009," CA Cancer Journal for Clinicians, vol. 59, no. 4, pp. 225-249, 2009.

[28] K. Imai, S. Hirata, A. Irie et al., "Identification of a novel tumor-associated antigen, cadherin $3 / \mathrm{P}$-cadherin, as a possible target for immunotherapy of pancreatic, gastric, and colorectal cancers," Clinical Cancer Research, vol. 14, no. 20, pp. 64876495, 2008.
[29] K. Imai, S. Hirata, A. Irie et al., "Identification of HLA-A2restricted CTL epitopes of a novel tumour-associated antigen, KIF20A, overexpressed in pancreatic cancer," British Journal of Cancer, vol. 104, no. 2, pp. 300-307, 2011.

[30] J. H. Sampson, A. B. Heimberger, G. E. Archer et al., "Immunologic escape after prolonged progression-free survival with epidermal growth factor receptor variant III peptide vaccination in patients with newly diagnosed glioblastoma," Journal of Clinical Oncology, vol. 28, no. 31, pp. 4722-4729, 2010. 


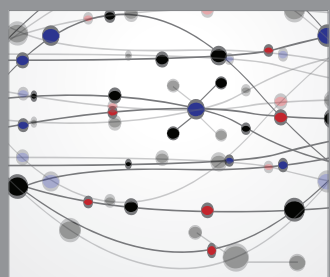

The Scientific World Journal
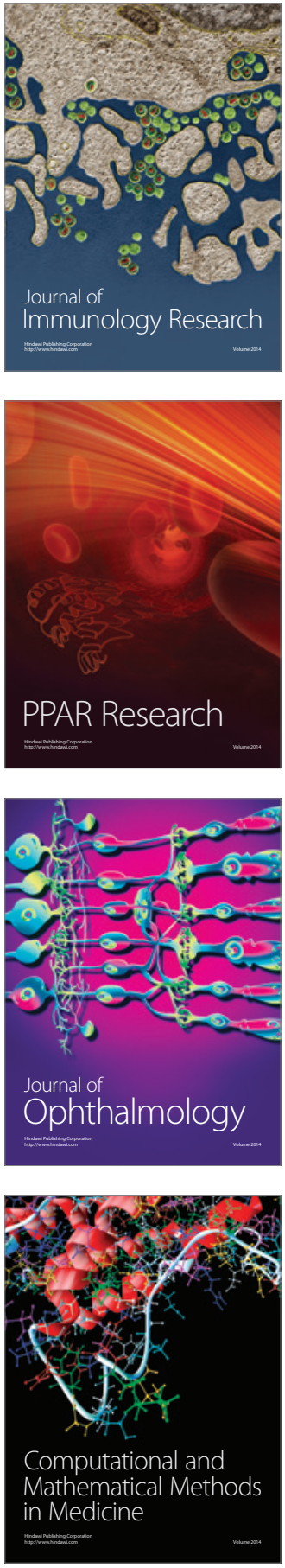

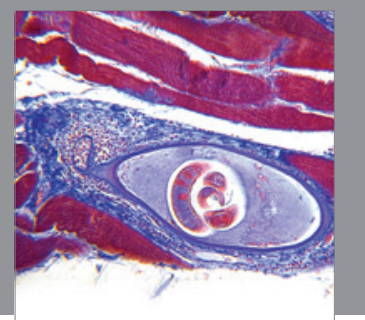

Gastroenterology

Research and Practice
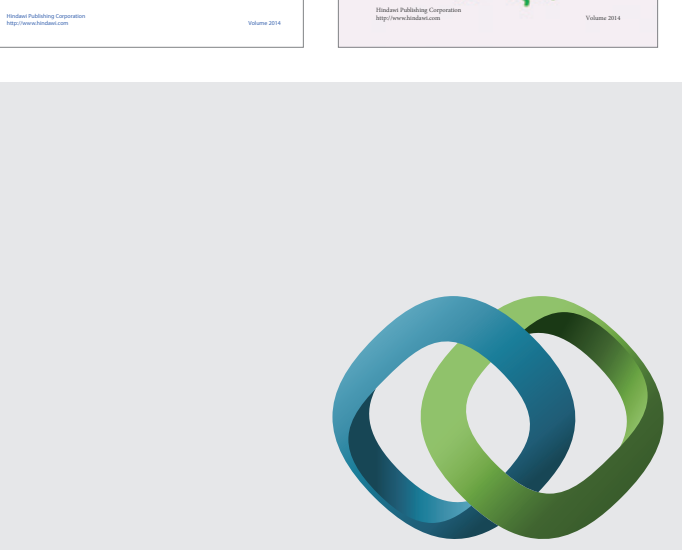

\section{Hindawi}

Submit your manuscripts at

http://www.hindawi.com
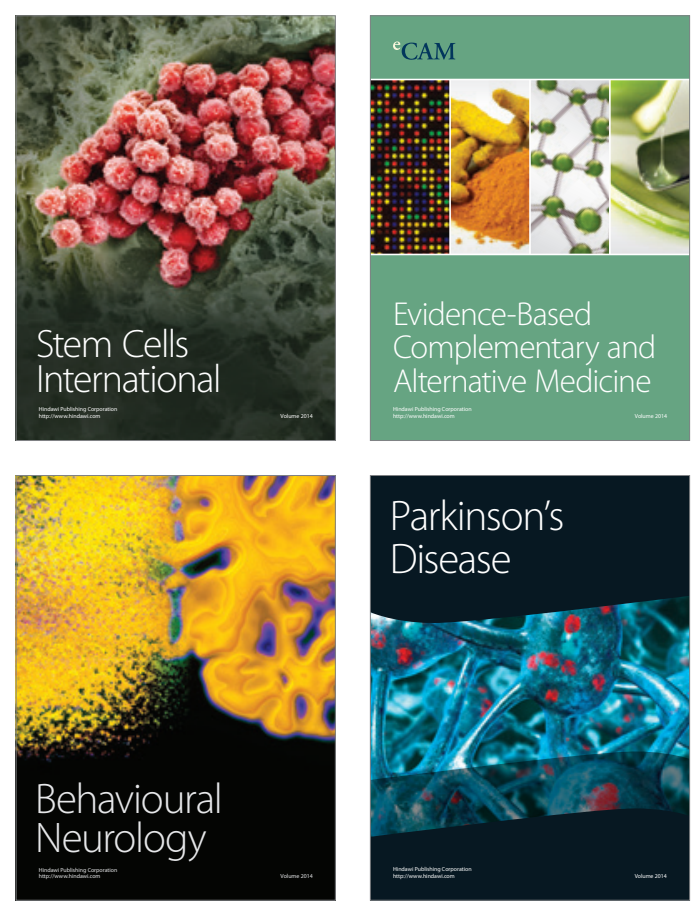

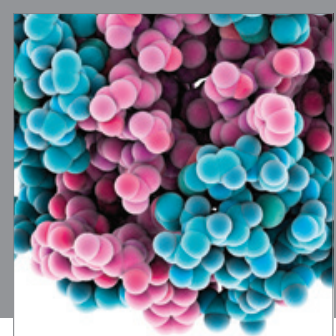

Journal of
Diabetes Research

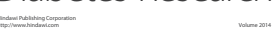

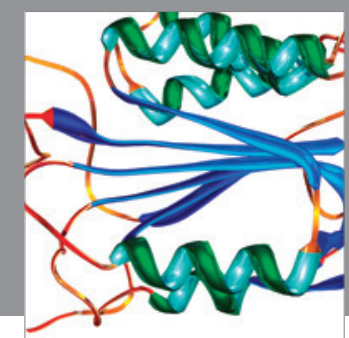

Disease Markers
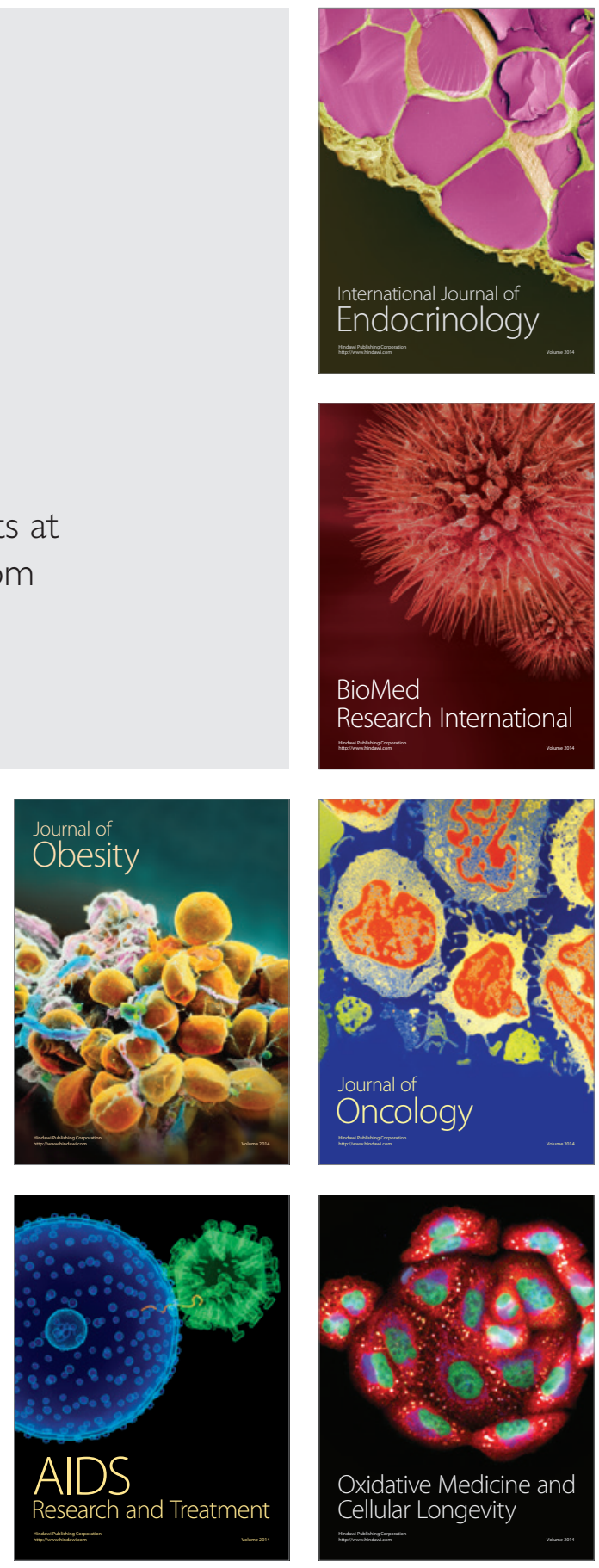